\title{
Gestión académico-administrativa en la educación básica
}

\author{
Inciarte, Alicia* \\ Marcano, Noraida** \\ Reyes, María Elena* ${ }^{\star \star *}$
}

\section{Resumen}

Esta investigación estuvo dirigida a analizar los procesos que conforman la gestión académica y administrativa en las esuelas básicas de la región zuliana adscritas a la administración pública. Los fundamentos teóricos se corresponden con el estudio de los componentes y subcomponentes que constituyen dicha gestión. La metodología utilizada, responde al tipo de investigación descriptiva-explicativa en el marco de un diseño no experimental, transversal y de campo. Se trabajó con una muestra de 854 docentes de aula y 161 directores y subdirectores; la recogida de datos se realizó con dos cuestionarios. Esta data se procesó con el modelo de análisis factorial y estadística descriptiva. La información cualitativa fue recolectada, mediante talleres realizados con docentes; estos permitieron develar situaciones y carencias que complementaron la explicación del objeto de estudio. Los hallazgos generados en la investigación señalan, que existen diferencias en la estructura material, académica y administrativa en las escuelas básicas, de hecho, se observan instituciones educativas con alto y mediano desempeño en zonas urbanas, encontrándose en los espacios geográficos rurales y urbanos marginales escuelas cuyas carencias y limitaciones no le permiten acercarse a un desempeño aceptable.

Palabras clave: Gestión académico-administrativa, educación básica, procesos curriculares y organizacionales.

* $\quad$ Profesora Titular de la Universidad del Zulia. Doctora en Educación. PPI Nivel III.

E-mail: ainciart@telcle.nte.ve.

** Profesora Titular de la Universidad del Zulia. Doctora en Ciencias de la Educación. PPI Nivel II.

E-mail: noraespina@hotmial.com.

*** Profesora Titular de la Universidad del Zulia. Doctora en Ciencias Humanas. 


\section{Academic-administrative management in Basic Education}

\section{Abstract}

This research project was focused on analyzing the processes constituting academic and administrative management in basic schools registered with public administration in the region of Zulia. The theoretical bases correspond with a study of the components and subcomponents making up said management. The methodology used was a descriptive-explicative type of research within the framework of a transversal field study with a non-experimental design. A sample of 854 classroom teachers and 161 directors and subdirectors was used; data was collected using two questionnaires. This data was processed using the model of factorial and statistical descriptive analysis. Qualitative information was collected using workshops carried out with the teachers; these allowed for revealing situations and deficiencies that complemented the explanation of the purpose of the study. Findings generated by this investigation indicate that differences exist in the material, academic and administrative structures in basic schools; some educational institutions with high and medium performance levels were observed in urban zones, while rural and marginal urban geographic spaces had deficiencies and limitations that did not allow them to approach an acceptable performance level.

Key words: Academic-administrative management, basic education, curricular and organizational processes.

\section{Introducción}

En el presente estudio se exponen las principales ideas en torno a la gestión académica y administrativa de la Educación Básica, a los procesos que la conforman y hacen posible la práctica pedagógica en la que se inscribe el proceso de enseñanza y aprendizaje.

Las políticas educativas del Estado venezolano, desde la década de los ochenta hasta el presente, se han orientado con mayor fuerza al nivel de Educación Básica, impulsadas por la problemática interna del nivel, ante la necesidad de actualización del personal docente y mejora de sus procesos, estructura y funcionamiento, bajo la consideración de que la Educación Básica es un espacio socio-educativo prioritario para ampliar la cobertura y la atención a la mayor cantidad de población en edad escolar, con necesidades apremiantes de formación.

En este contexto, las organizaciones de Educación Básica, en especial las del sector oficial, presentan problemas en la gestión académica y administrativa, (Pérez, 2000; Altuve, 1997; Bravo et al., 2000), que afectan los procesos curriculares y organizacionales e inciden de forma negativa en la práctica pedagógica.

Discutir sobre la gestión académica y administrativa refiere a una serie de procesos curriculares que implican no sólo los de enseñanza-aprendizaje desarrollados en el aula, sino también la gestión de los procesos administrativos que realiza la escuela para lograr los objetivos de la educación. De allí que, la gestión debe ser asumida como una totalidad en la que todos sus elementos se articulen en una red a través del recorrido que 
abarca la praxis curricular en la organización escolar. De no considerarlo así, se podría incurrir en la conducción de una gestión definida por la desarticulación y por una visión fragmentada de sus distintos componentes.

Esta situación, plantea exigencias al personal docente: directivo y de aula, en cuanto a la necesaria posesión de conocimientos, competencias y disposición para un mejor desempeño en sus funciones, orientadas al mejoramiento de la calidad del servicio educativo, en beneficio de la formación integral del estudiante que asiste a las escuelas básicas.

Para lograr la coherencia y pertinencia de la gestión académica y administrativa, es necesario desplegar una estrategia de control, vigilancia y seguimiento; junto a acciones de orientación y apoyo en cada uno de los procesos y actividades que se llevan a cabo. De modo que, no se produzcan desviaciones o distorsiones que obstaculicen el logro de los objetivos que dan razón a las acciones emprendidas en los ámbitos escolares e impidan elevar la calidad en la Educación Básica.

\section{La gestión académica en la educación básica}

Hacer referencia a la gestión en el marco institucional, amerita definirla en el contexto en el que ella se sitúa; "la gestión incluye la acción y el efecto de administrar de manera tal que se realicen diligencias conducentes al logro apropiado de las respectivas finalidades de las instituciones" (Palladino y Palladino, 1998:9).
Este planteamiento implica atribuir especificidad al término gestión en el escenario académico de la educación; en tal sentido, se denomina gestión académica al conjunto de procesos mediante los cuales se administran los diferentes componentes y subcomponentes curriculares que apoyan la práctica pedagógica en el continuo que permite construir y modelar el perfil deseable del estudiante.

En este orden de ideas, la gestión académica cubre un recorrido que involucra las acciones de atención al estudiante desde el ingreso hasta su desarrollo y egreso del sistema. En este recorrido, la acción de evaluación cumple un papel fundamental cuyo propósito y compromiso esencial es lograr que este proceso se cumpla dentro de lo previsto en lo académico, siempre dentro de las exigencias técnicas, organizacionales y las derivadas de las demandas del entorno.

La gestión académica se relaciona con los procesos de la realidad socioeducativa, entendida como el conjunto de interacciones dinámicas y complejas, desde las cuales emergen las situaciones didácticas que dan sentido a la práctica pedagógica. En esta gestión, se destaca la acción del docente como mediador de los aprendizajes, rol de investigador en proyectos educativos, rol de promotor y su gestión comunitaria. Igualmente surge como componente fundamental el aprendizaje de los alumnos, en este proceso el docente cumple una función vital como agente facilitador del desarrollo de competencias, articulado al rol de evaluador de los aprendizajes. 


\subsection{Roles del docente en interacciones dinámicas de la praxis curricular}

\subsubsection{Mediación de los procesos de aprendizaje}

Este rol involucra acciones dentro y fuera del aula, que ameritan de la participación y aportes de los docentes y alumnos, forman parte de la acción de mediación los medios y los recursos didácticos para la ejecución de los proyectos y la evaluación de los procesos y resultados generados en la acción educativa. En este rol el docente es un mediador entre los alumnos y el contexto, su papel es orientar e incentivar a los estudiantes para que desarrollen competencias, con capacidades para interiorizar los diferentes elementos que intervienen en el proceso educativo; el docente como mediador facilita la interacción para que el grupo participe en actividades de análisis y síntesis sustentadas en una acción reflexiva sobre lo realizado y lo que se puede realizar.

El docente como mediador tiene que estar en capacidad de proporcionar elementos conceptuales, procedimentales y actitudinales a los alumnos desde su posición de enseñar a pensar y aprender a aprender, a fin de apoyar en la construcción del conocimiento y en la realización de actividades que favorezcan el desarrollo del perfil de competencias esperado, todo esto, en función de las demandas que surgen de las múltiples y cambiantes situaciones del entorno, de esta forma participa en la configuración de procesos curriculares, dentro de metodologías integradoras y específicas estrategias de aprendizaje.
El docente como mediador atiende a los distintos tipos de conocimiento que implica el aprendizaje autónomo: (a) Conocimiento declarativo sobre los procesos de aprendizaje, el cual lleva implícito un conocimiento conceptual de los contenidos a dominar. (b) Conocimiento procedimental referido a cómo llevar a cabo los procesos requeridos para un dominio operacional, que es en esencia un contenido cognitivo y psicomotor. (c) Conocimiento actitudinal sustentado en contenidos sobre los valores, intereses y eticidad que guiarán los procesos.

En la enseñanza, la mediación fortalece los vínculos socioemocionales, morales y cognitivos para observar, comprender y mejorar el aprendizaje. En los procesos de mediación, la actividad del docente se desplaza hacia el alumno, eje central de la acción; el saber hacer de la acción se concreta por el aprendizaje de técnicas a través de experiencias, talleres, simulaciones, ensayo de trabajo colaborativo, entre otros.

La mediación implica la inducción a la acción a través de vivencias interactivas en la involucración de los estudiantes en procesos de aprendizaje; planteo de respuestas a través de interrogantes propuestas por el alumno para su resolución; todo ello, en atención a que la mediación facilita la aproximación al objeto de estudio mediante el desarrollo de experiencias, desde los cuales lo asimilado y discutido es mejor aprendido, a través del análisis y uso diario de nuevos elementos en el aprendizaje se pueden alcanzar niveles más elevados de conocimiento.

En opinión de Bar (1999), el educador ya no es el único poseedor de los conocimientos y el responsable exclusivo 
de su transmisión y generación, sino que debe asumir la función de dinamizador en la incorporación de contenidos por lo que sería conveniente mucho más amplio al correspondiente a su área o disciplina.

\subsubsection{El docente investigador en la ejecución de proyectos educativos}

El rol de investigador implica la búsqueda de información a todo nivel: docentes, alumnos, padres y representantes en actividades de revisión de materiales y discusión de su pertinencia en los proyectos planteados. La interacción constructiva sobre los contenidos tratados conlleva al análisis, interpretación y cotejo en el proceso de trabajo hacia la integración de los diversos elementos que permiten conformar el proyecto como producto.

Los proyectos educativos generados en el aula obligan al docente a considerar la investigación como actividad práctica orientada a la búsqueda de conocimientos ya establecidos a fin de conocer más sobre un hecho concreto que permita el planteo de nuevas visiones, adecuación a un contexto, resolución de problemas, e introducir cambios y sumar esfuerzos para que estos se concreten. Según Corrales y Jiménez (1994), el abordaje y concreción de proyectos educativos en el aula, demandan a los docentes convertirse en investigadores de su propio quehacer cotidiano, en especial si asumen una actitud crítica y experimental con respecto a su trabajo en el aula. Sin embargo, el docente necesita apoyo en el intento de generar una nueva cultura de investigación en la escuela. Esta acción de investigación puede facilitar el cambio de actitud del docente hacia su trabajo escolar.

La generación y concreción de proyectos en el aula permiten dinamizar y sintetizar contenidos curriculares, se convierte en una tarea dinámica para todos los actores del proceso educativo: docentes, alumnos, personal directivo, padres y representantes, quienes giran en torno a un centro de interés, el proyecto educativo, a partir del cual la acción investigativa debe centrarse en la interacción, utilizando preguntas, respuestas, cambios de tema, comentarios evaluativos, entre otros.

La acción de investigación conjuga el ser, conocer, hacer y convivir en un proceso dividido en fases que implican familiarizarse con la información, revisiones constantes, discusión de temas, cambios de acción y de rutina, se generaliza la participación y se acoge el proyecto por parte del colectivo; entre todos revisan las regulaciones o normas, acuerdos y reglas, entre una actividad y otra.

En opinión de Corrales y Jiménez (1994: 78):

"Los docentes investigadores reconocen que el proceso de enseñanza-aprendizaje es muy complejo, en el cual intervienen distintas variables que interactúan, al conocer estas variables los educadores investigadores pueden realizar acciones en el proceso enseñanza-aprendizaje de forma sistemática. De manera que estos docentes se puedan dar cuenta de que uno de los objetivos de la investigación en el aula, es documentar el modo en que ellos enseñan y en el que los estudiantes aprenden". 
Los proyectos de investigación educativa permiten al docente desarrollar competencias para la indagación socioeducativa, además de la oportunidad de observar, reflexionar, hacerse preguntas e interpretar la información, generándoles conocimientos en su desarrollo profesional y mejoramiento en los procesos de enseñanza-aprendizaje. Contreras (1995: 35) señala que, "un primer paso hacia la vinculación de la reflexión teórica con la práctica que se desarrolla en las aulas debe considerar la necesidad de ofrecer oportunidades y espacios para que los docentes reflexionen acerca de la práctica".

\subsubsection{El rol de la difusión desde la organización comunitaria}

Este rol está vinculado a los de mediador, investigador, evaluador; el mismo se hace presente desde el primer momento con la acción de mediación, cuando se inicia el planteo y búsqueda de materiales con información, entrevistas y conversaciones con personas destacadas o conocedoras en esa área del saber que se indaga. Igualmente está asociada a la investigación cuando se toman decisiones sobre qué información se toma para la construcción de ese marco teórico conceptual que se construye con relación al proyecto. Cuando el proyecto se presenta y se exponen al público los resultados, se hace manifiesta la acción difusiva como tal.

La preocupación del sector educativo se ha orientado en sus postulados teóricos hacia el fortalecimiento de la gestión participativa del docente en las escuelas en sus relaciones intra e interinstitucionales, este interés por extender esta acción al entorno se acentúa ante la necesidad de cobertura de la población escolar. La cercanía entre la organización educativa del nivel de educación básica y la comunidad, forma parte de la filosofía de este nivel que se moderniza bajo una perspectiva más social.

La acción difusiva desde la organización comunitaria fortalece la acción social de la escuela, amplía su proyección hacia la comunidad, permite intercambio desde la cultura organizacional escuelacomunidad resurgiendo, de esta manera, una vinculación más estrecha entre estos dos ámbitos, haciéndose partícipes de sus propios problemas, potencialidades y limitaciones, buscando su propia transformación a partir de una perspectiva más crítica, social y moral que facilite el recate de valores que guíen las acciones por caminos más éticos hacia el logro de una equidad social que equilibre tantas desigualdades que, en cierta forma, también se reflejan en la escuela.

Las actividades de difusión pueden ayudar a una mayor apertura y democratización desde la escuela y a más amplia proyección social de su praxis pedagógica. La acción de difusión desde la escuela da oportunidades a los miembros de la organización para familiarizarse con los problemas del medio, entrar en contacto con la comunidad y devolver a ésta un servicio de calidad. Actualmente, es fundamental proyectar la labor del docente en la comunidad para reforzar los procesos de aprendizaje, no solo de los estudiantes, sino de los propios padres y de la familia en general, la cual avala la función social de la escuela; el propósito es facilitar los cuatro aprendizajes propuestos por Delors (UNESCO, 1996): aprender a conocer, aprender a ser, aprender a ha- 
cer y aprender a convivir, implica también extender estos conocimientos a la comunidad.

Mediante la acción difusiva se amplía la influencia y promoción de la escuela a fin de que responda al momento histórico y al perfil que se aspira en el conjunto de actividades orientadas a un autofortalecimiento articulado con la comunidad en que actúa e integrando las diversas fuerzas que la componen en una línea de pensamiento que conjuga un sentido prioritario: educar para mejorar.

La escuela básica está llamada a elevar los niveles espiritual, intelectual, moral y técnico en la formación para el trabajo de los grupos sociales, no como una proyección de la escuela a la comunidad, sino en común con ésta, lo cual implica integrar a la familia, la escuela y la comunidad en una visión y acción compartidas en función de sus miembros que demandan una vida más equitativa y segura; es sobre este punto que la acción de difusión puede ser concretada y ayudar a generar un proceso de participación y cooperación.

\subsubsection{Aprendizaje y desarrollo de competencias}

En la gestión académica, es necesario hacer una revisión de sus procesos, específicamente el de la interacción social entre docentes, alumnos y demás miembros de la comunidad educativa y en relación a los contenidos, lo cual conduce al aprendizaje del alumno. En opinión de Florez (1999: 22) "El enseñar y el aprender son procesos correlativos, no puede confundirse el uno con el otro, pues el aprendizaje ocurre al interior de cada sujeto que aprende, es subjetivo, aunque su dominio puede exteriorizarse eventualmente en palabras y acciones específicas, este concepto está restringido al ser humano". Aunque es innegable el carácter individual y endógeno del aprendizaje escolar, éste se compone no solo de representaciones personales, sino que se sitúa en el plano de la actividad social y la experiencia compartida.

El aprendizaje es un proceso que tiene como punto de partida la situación del alumno, sus intereses, necesidades aspiraciones y los objetivos que desde ellas es posible plantear. El proceso de aprendizaje ha sido tradicionalmente un objeto de interés muy importante para los especialistas de la educación; sin embargo, resulta difícil ponerse de acuerdo sobre el enfoque mas adecuado para acercarse a su estudio en relación al que aprende como alguien a quien la escuela ha de enseñar a pensar, o mejor, como alguien que puede aprender a pensar. En cualquier caso, aprender a procesar información activamente y de modo que resulte significativa a la vez que aplicable a la vida práctica, no es tarea fácil.

El aprendizaje y las habilidades de razonamiento se desarrollan no como mecanismos abstractos, sino como procesos que permiten procesar determinados conocimientos en contextos específicos y con metas específicas. El alumno, en su aprendizaje, se enfrenta a diferentes tipos de problemas que imponen situaciones nuevas, en los que debe codificar, comparar, combinar nuevos tipos de información; de esta manera, se facilita en el que aprende la interacción a partir del conocimiento que ya posee, así como trasladar el pensamiento a la acción. De esta forma, los que aprenden han de ser capaces de transferir su conocimiento a 
través de sus habilidades y destrezas a situaciones de la vida real.

Es fundamental, en el contexto educativo, se fijen metas en relación al abordaje y sistematización del conocimiento; en tal sentido, es necesario considerar las estrategias que aplica el docente para organizar, procesar y actualizar una determinada información. Las estrategias pueden relacionarse también con procesos de control del desempeño, tanto el docente como el alumno, pueden controlar y hacer seguimiento al proceso de aprendizaje.

En los procesos de aprendizaje, es fundamental determinar cuáles pueden ser las mejores estrategias para facilitarle al estudiante el desarrollo de las competencias requeridas en relación a los objetivos y al perfil deseado. En tal sentido, se reconoce la importancia al respecto, dado que las habilidades, destrezas, valores y actitudes en su desarrollo, suponen la interdependencia con estructuras y organización, con conocimientos previos que contribuyen a la asimilación y comprensión para la elaboración de nuevos saberes.

\subsubsection{La praxis curricular}

La educación se ha constituido siempre en eje prioritario para la sociedad por el relevante papel que cumple en la formación del ser humano. En este marco, aparece el currículo como referente teórico, metodológico y práctico que da sentido a la educación como proceso organizado e institucionalizado a través del cual se concreta la cultura como manifestación que le asigna identidad y particularidad desde el contexto en el que se desarrolla.

En este sentido, el currículo se constituye en el elemento estructurador de las diferentes interacciones que se establecen desde sus diversos componentes en el proceso enseñanza-aprendizaje; a través de él, se organizan, desarrollan y evalúan las actividades y momentos de ese proceso. El currículo no es un concepto etéreo, es un proyecto educativo que orienta las múltiples facetas que conforman la práctica pedagógica en torno a la organización del conocimiento y de las actitudes y valores que expresan el docente y los alumnos.

La praxis curricular emerge hoy como una acción más compleja, ya que se espera que las escuelas proporcionen una amplia gama de posibilidades que faciliten el manejo de contenidos conceptuales, procedimentales y actitudinales hacia el desarrollo de habilidades, destrezas, valores y actitudes que permitan razonar y actuar eficazmente, acordes a las necesidades y demandas del entorno. Es así, como los contenidos curriculares contribuyen al perfilamiento de las competencias académicas y actitudes valorativas.

El currículo actual plantea un cambio de perspectiva que se refleja en un proceso en el que se evidencia la necesidad de socializar la gestión del currículo a partir de una perspectiva de negociación que atienda las diferentes inquietudes $y$ necesidades de los diversos grupos que intervienen en el proceso educativo, en virtud de la misión y visión de la organización educativa.

La adecuada gestión del currículo, conlleva a considerarlo como un instrumento que viabiliza la educación y la justifica como servicio y acción social prioritaria para el desarrollo humano en toda su extensión. Según Wilson (1992: 16-20). 
"El currículo constituye una amplia gama de habilidades prácticas, sociales y personales necesarias para la vida y reaccionar creativamente ante las oportunidades de la sociedad moderna, en el intento de darle pertinencia al currículo, estructurado en función del alumno".

El autor antes citado, al hacer referencia a las prácticas educativas, considera que muchos de los responsables de la gestión de las escuelas han desarrollado nuevos diseños curriculares sin considerar modelos didácticos, recursos instruccionales y técnicas de evaluación adecuadas. Es necesario entonces, un hacer más reflexivo en torno a los elementos que conforman el currículo, en un compartir intra e interinstitucional orientado a generar un nuevo comportamiento en el personal dentro de la organización.

Según Romero (1996: 89)

"Los propósitos y los fines de la educación se concretan, entre otros, en los centros educativos por parte del profesor en el aula. De ahí la importancia de analizar la cultura organizacional y la gestión de la educación, elementos estos que dinamizan la cotidianidad de la escuela y de las aulas".

La gestión del currículo y de sus resultados es responsabilidad directa del personal directivo, docente y demás miembros de la comunidad educativa de acuerdo a los diferentes grados de participación de estos actores. Del estilo de dirección establecido en la escuela, depende la dinámica de las acciones desarrolladas; de allí, la importancia del liderazgo compartido, para contribuir a elevar los niveles de calidad de la organización y el logro de las metas y objetivos.

\subsubsection{La Práctica Pedagógica}

La práctica pedagógica hace referencia al acto asociado a la red de relaciones materiales, espaciales, temporales, institucionales, estructurales y organizacionales que condicionan la orientación de las interacciones, procesos y resultados atendidos desde la gestión académica. Esta práctica, en sentido amplio, se puede definir como el conjunto de elementos materiales, instruccionales o didácticos y relacionales que se articulan en una dinámica estructural y funcional que se concreta en el proceso de enseñanza-aprendizaje, la misma se constituye en el núcleo esencial de la gestión académica y es la que le imprime unidad desde la diversidad que caracteriza al proceso educacional.

En sentido específico, la práctica pedagógica se inscribe en el mundo de la clase, la cual se entiende como el conjunto de actividades que están determinadas por los objetivos generales y específicos, las áreas de conocimiento, los bloques de contenidos, ejes transversales y proyectos pedagógicos desarrollados a partir de un entorno que se estructura con objetos materiales y la organización de los actores sociales que participan directamente en las situaciones de aprendizaje, en un marco de acciones e interacciones interdependientes donde concurren acontecimientos particulares que signan, con especificidad, el proceso de la clase en cada uno de sus momentos.

Según Vayer, Duval y Roncin (1993: 17) "En educación, la clase es un conjunto de niños de la misma edad, reunidos para a recibir colectivamente una enseñanza bajo la dirección de un adulto. 
También se refiere al lugar o aula donde se reúnen". Sin embargo, en la concepción actual de la educación, a la clase se le representa con mayor diversidad en relación a las condiciones, características y ritmos de aprendizaje de los estudiantes, así como a los contenidos, metodologías y formas de evaluación, entre otros aspectos; donde el alumno se convierte en el principal protagonista en función del significado que para él tienen las actividades dentro del contexto material y relacional en el que se ubica la clase $y$, en donde el docente lleva a cabo la gestión del proceso enseñanza-aprendizaje.

La clase es, entonces, la unidad funcional de la práctica pedagógica, pues desde ella se produce la construcción de la realidad educativa, es un espacio abierto de confluencias y emergencias personales, sociales y educativas en la que se pueden expresar contradicciones diversas, que aun cuando algunas de ellas se originen fuera de su campo, repercuten en su interior. Esto implica que, la práctica pedagógica reviste cierta complejidad dados los intereses, necesidades, expectativas, motivaciones, conflictos y resistencias que allí afloran, lo cual da origen a comportamientos que, en algunos casos, interfieren con el orden de los procesos.

Cuando eso ocurre, se le dificulta al docente mantener el control de las actividades por la singularidad o peculiaridad de la actuación de los estudiantes, en esta situación influyen las desigualdades sociales, cognitivas, actitudinales y procedimentales; porque es en el transcurrir de la práctica pedagógica donde se develan también los conflictos respecto al aprendizaje.
La interacción entre el docente y los alumnos está basada en las vivencias y experiencias a partir de las condiciones y situaciones que se establecen y desde las cuales se difunden los valores bási$\mathrm{cos}$, se generan las actitudes y los componentes cognitivos, socio-emocionales-morales, relacionados con las decisiones que toma el grupo en la clase. En todo este proceso, las acciones de evaluación son fundamentales y, para ello, se deben instrumentar mecanismos conducentes a la obtención y registro de la mayor cantidad posible de información que, luego de ser procesada permita reorientar los cursos de acción para hacer efectivas y oportunas las respuestas que requieren las situaciones planteadas.

\section{La gestión administrativa en la educación básica}

La gestión administrativa se entiende como un sistema abierto, flexible, dinámico y continuo, conformado por procesos, componentes, subcomponentes y principios de gestión que orientan, equilibran y hacen posible el desarrollo del currículo a través de la realización de acciones dentro de ciertas normas, valores y procedimientos que permiten viabilizar los diferentes procesos para que éstos se cumplan tal como lo establece el marco legal, filosófico y teórico por el que se rigen las instituciones.

Desde la gestión administrativa se crean condiciones para prever, organizar, coordinar, controlar, monitorear y hacer seguimiento a los procesos que se generan en la ejecución de proyectos y acciones, racionalizando los recursos humanos, materiales y financieros, en aten- 
ción a los objetivos formulados. En el sistema educativo, la gestión administrativa se apoya en las teorías y prácticas propias del campo de conocimiento de la administración en general y de la administración escolar en particular. Esta gestión se sustenta en la filosofía de la institución, la cual se refleja en la misión, visión y objetivos que la guían y, a partir de ellos, se determinan la estructura y funcionalidad de la organización como tal.

La continuidad, coherencia y sistematización que caracterizan la gestión administrativa, vienen dadas por los procesos de planificación, organización, dirección y evaluación. La gestión está vinculada a estos procesos, de los cuales extrae los elementos que la hacen operativa y le dan el carácter dinámico que le es propio. En este sentido, Palladino y Palladino (1998: 9) plantean que:

"La gestión incluye la acción y el efecto de administrar, de manera tal que se realicen diligencias conducentes al logro apropiado de las respectivas finalidades de las instituciones. Etimológicamente gestión es el conjunto de actuaciones integradas para el logro de objetivos a largo, mediano y corto plazo. La gestión es la acción principal de la administración, es un eslabón intermedio entre la planificación y los objetivos que se pretenden alcanzar. Radica en activar y promover la consecución de un mandato o encargo. En general, la gestión comporta entre otras, acciones administrativas, gerenciales, de política de personal, económicas, de planificación, programación, orientación y evaluación."

\subsection{Procesos administrativos}

Los procesos administrativos comprenden fases o etapas a través de las cuales se instrumentan los cursos de acción requeridos por la gestión, a partir de los recursos y en atención a las políticas establecidas. Los procesos administrativos se encuentran en estrecha interrelación respecto a la totalidad de acciones dirigidas al logro de los fines y objetivos de la organización. A objeto de precisar la naturaleza e importancia de los mismos, se expondrán brevemente cada uno de ellos, así como algunos de los elementos que les tipifican.

\subsubsection{Planificación}

La planificación es un proceso fundamental de la gestión administrativa, implica ante todo previsión, parte del diagnóstico de necesidades, formulación de políticas, misión, visión, objetivos, fijación de metas y previsión de recursos; estos elementos son primordiales y responden al contexto de la institución para la cual se planifica, en consideración a ello, se definen los cursos de acción y se seleccionan los medios para llevar acabo las acciones que permiten lograr los objetivos. Sobre este punto Diez et al., (2001:5) afirman que, "la planificación constituye la función más importante del proceso administrativo. Es esencial para que las organizaciones logren objetivos y mejores niveles de rendimiento, incluye elegir y fijar las misiones y objetivos de la organización".

En esa misma línea de pensamiento, Bounds y Woods (1999: 90-93) señalan que: 
"La planificación es decisiva, es el proceso mediante el cual organizaciones e individuos tratan de pronosticar el futuro, anticipar problemas, identificar oportunidades y aprovechar plenamente el personal, tiempo y otros recursos. Representa un primer paso esencial para hacer mejoras en toda la organización."

La planificación implica una estrategia encadenada de acciones en relación a acontecimientos cotidianos, para fijar dirección y establecer cierto margen de seguridad a los cambios necesarios que se deben hacer; se sustenta en acciones adecuadas para que cualquier intento de cambio aporte resultados positivos con un mínimo de dificultades. Se le puede definir como un proceso sistemático y continuo que parte de acciones pasadas para perfilar y anticipar nuevas estrategias y escenarios posibles, mediante ella se busca responder a los retos que se le plantean a las instituciones respecto a las exigencias sociales.

En este sentido, la planificación es un proceso crucial en la gestión administrativa, con respecto a la utilización adecuada de los recursos existentes para lograr, desde una perspectiva racional, estratégica y prospectiva, la construcción de escenarios para el inicio, desarrollo y consolidación de acciones pertinentes a los requerimientos del contexto interno y externo de la escuela.

\subsubsection{Organización}

El término organización hace referencia a un sistema orientado a formalizar los comportamientos en los desempeños $y$, a buscar esquemas generalizadores de motivación al logro, de determinados fines mediante la ordenación y disposición de elementos para dar cumplimiento a diversas funciones según la misión establecida. El vocablo organización se utiliza en diversos campos de la sociedad, en atención a la estructura y función que asume una entidad social, en sentido amplio, puede ser una empresa o corporación que aglutina a grupos de personas que interactúan con el fin de lograr los objetivos, también se refiere a un proceso de la gestión administrativa, en este contexto, se asocia a la acción de preparar, ordenar, estructurar, disponer de manera conveniente los recursos humanos, materiales y otros que sean pertinentes con los objetivos que le marcan horizonte.

La organización como proceso conlleva a un encadenamiento de acciones orientadas por el qué y cómo ha de hacerse, de modo que exista un método para la aplicación de procedimientos, estrategias y técnicas en la instrumentación de las operaciones. Implica la adecuada disposición de los elementos (medios, personas) necesarios para la conducción y mantenimiento de un sistema, en el que todos los miembros cumplan su contenido con eficacia y calidad. La organización lleva implícito un hilo estructurado que permite armonizar, acordar, ajustar y adecuar las interrelaciones entre el personal y las instancias de una institución para un mejor funcionamiento y equilibrio en la articulación de los componentes de la totalidad o sistema; como proceso tiene el propósito de asegurar la correspondencia entre las disposiciones oficiales y el ideario o proyecto que define la filosofía de la institución.

Mediante la organización se operacionalizan las políticas, misión, visión, objetivos, metas, estrategias y recursos que se plantea la institución, es lo que permite 
la realización de las diferentes funciones y hacen que se cumplan cabalmente. La organización facilita la diferenciación e integración de los distintos elementos, de modo que, se establezca la estrecha conexión con los mecanismos de estabilización, el rendimiento y permanencia del sistema.

En la organización es fundamental cuidar los recursos, disponerlos en el orden requerido para el desarrollo de los procesos y logros en los resultados. En este orden de ideas, el recurso humano se constituye en un elemento trascendental dada su estructura personal integrada por conocimientos, experiencias, motivaciones, emocionalidad, inquietudes, necesidades y expectativas, valores que lo configuran como un ser social que le permiten responder a una multidimensionalidad de factores (culturales, psicológicos, sociales, espirituales) que entran en juego en su interacción con otras personas, y a partir de la cual establece una dinámica compleja, primordial para atender desde la determinación de roles, sus funciones y tareas.

La organización del trabajo en los centros educativos conlleva a una acción deliberada y cuidadosa dado que constituye un entorno de aprendizaje en todas sus dimensiones. "Una escuela es un ambiente que un grupo de personas ordena para estimular el aprendizaje, y contribuir a la formación de los miembros que en él participan" (Amarante, 2000: 5).

La escuela puede ser definida como un sistema abierto en el que se articulan personas y medios en función de unos objetivos comunes y que inserta en un contexto determinado bajo la dirección del estado y la sociedad. En la escuela se esta- blece la estructura organizativa asociada a los fines, objetivos y metas que la guían mediante la formalización contenida en reglamentos, normas y procedimientos administrativos, determinación de funciones $y$ tareas y las interrelaciones entre las diferentes posiciones del personal.

La estructura formal de la organización escolar puede definirse como la integración de los modos en que el trabajo o servicio es dividido entre diferentes acciones o tareas coordinadas para lograr la consistencia interna del funcionamiento de la escuela. Sin embargo, en su interior se entrelazan la estructura formal y la informal (actuaciones espontáneas de grupos no previstas en la formal), esto le imprime una dinámica organizacional particular al centro escolar. La expresión de la organización formal puede observarse en los organigramas que reflejan gráficamente los elementos caracterizadores de la estructura institucional.

La organización escolar parte del conjunto de medios de que dispone para conducir el proceso educativo hacia la superación constante de las personas y los procesos que se atienden de acuerdo al currículo que se administra. A partir de la organización como proceso, el personal directivo se ocupa de los problemas materiales, relacionales, instruccionales, cognitivos y actitudinales que entrañan el gobierno y la gestión administrativa de la comunidad escolar. En este sentido, la dirección del plantel procura que la estructura y funcionalidad escolar facilite la optimización de los servicios educativos que ofrece a la población usuaria del sistema.

Organizar la actividad escolar implica, organizar el ámbito para la vida del estudiante, disponiendo de los recursos y 
estructurando situaciones para la expresión de los diferentes potenciales creadores y se sienta motivado, animado y apoyado en sus esfuerzos asociados a la adaptación, aprendizaje, construcciones y rendimiento; "no perseguido y ahogado por sistemas rígidos y autoritarios que matan toda iniciativa y que conducen a la rutina" (González, 2000: 58). El estudiante constituye el componente curricular de mayor fuerza, y es él, quien justifica los deliberados esfuerzos que se hacen para su formación.

\subsubsection{Dirección}

Dirigir una organización social, implica ponerla a funcionar a partir de una estructura organizativa para guiarla desde el sitio donde hoy se encuentra, hacia un lugar mejor en el futuro. La dirección es la expresión integrada entre recursos, estructura y estrategia, que se sintetizan en la aplicación de procedimientos y mecanismos en los procesos llevados a cabo. En este sentido, la dirección marca la orientación a la acción concreta y simultánea a la ejecución de las actividades; es el proceso de gestión que hace posible la planificación y la organización a través de la programación, desarrollo y monitoreo de los procesos de las personas que intervienen como responsables e implicadas en los mismos.

En el proceso de dirección cobra significativa importancia gestionar el recurso humano en cuanto a las relaciones interpersonales y el comportamiento de las personas, de manera que, se les facilite el reempeño de acuerdo a las funciones establecidas, a través de un proceso que concilie los intereses de los trabajadores y la organización y, canalice las necesidades, mantenga la disciplina y la unidad de dirección. Sobre este punto Lepeley (2001: 19-20) afirma que "la satisfacción de necesidades básicas, afectivas, económicas y espirituales, permiten cimentar las bases humanas y afectivas que son imprescindibles para implementar con éxito una gestión de calidad".

En el contexto de la educación, la dirección de la gestión académica ha de ser cuidadosamente manejada ya que requiere la revisión y actualización de sus procesos en forma permanente. Los directores tienen la mayor responsabilidad en la complejidad dinámica de la organización educativa.

Acerca de esta función, González (2000: 68) plantea que:

"La dirección escolar tiene carácter complejo, del director depende el acierto o fracaso de los planes y la organización de los mismos. Resulta difícil manejar personas, el director de toda institución educativa deberá cultivar y respetar al elemento humano en todas sus fases y manifestaciones, deberá tratarlo en un estado de autoestima que le permita el mejor rendimiento y disposición de elevar la calidad de la enseñanza, la de los alumnos y, con ello, el medio donde se desenvuelve".

Las ideas expuestas por el autor, remiten a las múltiples facetas que debería atender el director de una escuela en las interrelaciones sociales, exige conocimiento de las condiciones y situaciones en las que vive y se desenvuelve el personal a su cargo: docentes y alumnos; a fin de prestar atención a los aspectos humanos, conduciéndolos mediante un liderazgo flexible, creativo y diligente. Dirigir es, entonces, conducir correctamente la realización de actividades hacia el térmi- 
no señalado, ello incluye los siguientes aspectos: liderazgo, toma de decisiones, comunicación, trabajo en equipo y manejo de conflictos.

\subsubsection{Evaluación}

Al término evaluación se le confieren diferentes significados y puede aplicarse en escenarios, procesos y actores diversos y en variadas circunstancias. Su práctica se realiza a través de un conjunto de estrategias, procedimientos e instrumentos, siguiendo los lineamientos de un determinado modelo de evaluación o combinando electos teóricos, procedimentales de varios modelos con el objeto de obtener la información requerida para conocer las condiciones y situaciones en las que se llevan a cabo los procesos y se analizan los efectos de los mismos, al compararlos con las metas fijadas.

En tal sentido, en este proceso se consideran tanto las necesidades y limitaciones encontradas, como los niveles de logro alcanzados; de este modo, la evaluación facilita la confrontación de lo que se ha hecho con lo que se quería hacer y, con ello, la toma de decisiones a fin de mejorar la planificación futura. La evaluación como proceso de valoración ha ampliado su espacio y cualquier actividad, proceso, gestión, comportamiento y reempeño puede ser evaluado en los diversos ámbitos de operación y actuación de una organización social.

En los últimos años, la evaluación se ha convertido en un tema de interés en las instituciones económicas, políticas, culturales, sociales y educativas; en este último sector, ha trascendido "la evaluación centrada en el aprendizaje, o en el rendimiento del alumno. Hoy se habla de planes de evaluación, criterios de evaluación, evaluación de procesos, evaluación de resultados y evaluación del sistema educativo" (García, 1997: 191). Lo cual indica que, progresivamente se ha ido desarrollando una cultura de la evaluación; las investigaciones y la literatura existentes sobre evaluación educativa, son una muestra de ese cambio.

La evaluación como proceso de la gestión administrativa, tiene entre los elementos básicos que la definen: el control, el monitoreo y el seguimiento; específicamente, los insumos, acciones, procesos y resultados, producto de la dinámica organizacional.

\section{Estructura metodológica de la investigación}

\subsection{Diseño de la investigación}

El diseño de la presente investigación es de carácter no experimental, transversal y de campo (Hernández y et al., 2000), (Bavaresco, 1997) y (Hernández, 1994). La aplicación del método seleccionado se realizó en dos fases: (a) una fase empírica que se vincula a la recolección de datos observacionales; se responde, así a la búsqueda de las fuentes de información (docentes en cargos directivos y de aula en instituciones de Educación Básica, primera y segunda etapa), para extraer los hechos de interés vinculados a la gestión académica y administrativa de estas escuelas; (b) una fase explicativa de construcción conceptual asociada al análisis e interpretación de la información en estrecha relación con la teoría que sustenta el estudio. 


\subsection{Población de estudio}

Se seleccionaron dos poblaciones, la primera conformada por el personal Directivo: Directores y Subdirectores; la segunda, por los docentes de aula. Ambas poblaciones laboran en la I y II etapas de los planteles de Educación Básica, adscritos a dependencias de la administración pública; específicamente las pertenecientes a la Zona Educativa (denominadas Escuelas Nacionales) y a la Secretaría de Educación del Estado Zulia (denominadas Escuelas Estatales), ubicadas en los siguientes municipios del Estado Zulia: Maracaibo, San Francisco, Jesús Enrique Lossada, Mara, Páez, Machiques de Perijá, Rosario de Perijá, La Cañada de Urdaneta y Sucre.

\subsection{Selección de la muestra}

La muestra tiene carácter intencional no probabilística; por cuanto se consideró como muestra al grupo de docentes: en cargos directivos y de aula (Tabla 1).

\subsection{Técnicas e instrumentos para la recolección de la información}

Los instrumentos de investigación aplicados fueron dos cuestionarios; el primero, (cuestionario A) constituido por 65 ítems, se orientó a determinar las acciones que desarrollan los docentes de aula, refiere al objetivo: Analizar la acción del docente como mediador, evaluador, investigador y promotor en la gestión académica requerida por el currículo básico nacional; este instrumento se elaboró en atención a la categoría de análisis gestión académica en la educación básica, conformada por las propiedades roles del docente en interacciones dinámicas de la praxis curricular; procesos del aprendiza- je en la práctica pedagógica; manejo de contenidos; componentes curriculares que apoyan la sistematización del proceso instruccional; lineamientos de apoyo al currículo; socialización del currículo en los procesos de escolarización.

El segundo cuestionario (cuestionario B) conformado por 63 ítems, dirigido a analizar las acciones administrativas que desarrolla el personal directivo en atención a los cambios curriculares propuestos en la educación básica. El mismo se orienta por la categoría de análisis gestión administrativa de la educación básica y la subcategoría procesos administrativos integrada por las propiedades planificación, organización, dirección y evaluación.

\subsection{Procesamiento de la información para la obtención de los Resultados}

La información se analizó a través del análisis factorial, éste es un modelo de agrupación de variables estadísticas o factores; este tipo de análisis facilita el estudio e interpretación de un conjunto de variables aleatorias, agrupándolas en uno o varios factores que las explican (Sierra, 1981; De la Garza, 1995; Brown, 1996; Escalona et al., 2000). En opinión de De la Garza (1995: 35) "el análisis de los factores (...), es una técnica que a partir de guías (criterios), conduce hacia donde está la respuesta". Al aplicar este procedimiento a la muestra del estudio (docentes de aula y personal directivo), se escogió el modelo de componentes principales con rotación varimax, y una vez obtenida la solución inicial (matriz), para determinar el número de factores, se utilizó el criterio de la raíz latente, que consiste en retener en la solución, sola- 
Tabla 1

Distribución de la muestra por dependencia, municipio, personal directivo y de aula

\begin{tabular}{|c|c|c|c|c|}
\hline \multirow{3}{*}{$\begin{array}{l}\text { Estado Zulia } \\
\text { Municipios }\end{array}$} & \multicolumn{4}{|c|}{ Administración Pública } \\
\hline & \multicolumn{2}{|c|}{ Zona Educativa Zulia } & \multicolumn{2}{|c|}{$\begin{array}{c}\text { Secretaría de Educación } \\
\text { del Estado Zulia }\end{array}$} \\
\hline & $\begin{array}{l}\text { Personal } \\
\text { Directivo }\end{array}$ & $\begin{array}{l}\text { Personal } \\
\text { Docente }\end{array}$ & $\begin{array}{l}\text { Personal } \\
\text { Directivo }\end{array}$ & $\begin{array}{l}\text { Personal } \\
\text { Docente }\end{array}$ \\
\hline Maracaibo & 39 & 280 & 19 & 140 \\
\hline San Francisco & 13 & 58 & 7 & 22 \\
\hline Jesús enrique Losada & 11 & 16 & 00 & 00 \\
\hline Machiques de Perijá & 1 & 72 & 3 & 26 \\
\hline Rosario de Perijá & 3 & 22 & 1 & 16 \\
\hline La Cañada de Urdaneta & 5 & 22 & 3 & 19 \\
\hline Mara & 16 & 55 & 7 & 39 \\
\hline Páez & 10 & 17 & 2 & 5 \\
\hline Sucre & 5 & 15 & 5 & 30 \\
\hline TOTAL & 114 & 557 & 47 & 297 \\
\hline
\end{tabular}

Fuente: Zona educativa Zulia (2003). Secretaría de Educación del Estado Zulia (2003).

mente los factores cuyos valores propios o autovalores son mayores que uno.

\section{La gestión}

\section{académico-administrativa en el contexto de la Educación Básica}

Los resultados del proceso de recolección de información se presenta en la Tabla 2, que sigue.

Del análisis de los procesos de la Gestión Académica y Administrativa de la Educación Básica, se infiere que, en relación al rol de mediador de los aprendizajes, los docentes de aula estructuran situaciones didácticas y crean condiciones para que los educandos participen en las diversas situaciones vinculadas a los procesos del aprendizaje, además, les ayu- dan a tomar conciencia de sus potencialidades, cuestión que confirma la atención prestada por los docentes a los requerimientos planteados, en el marco teórico y metodológico del Currículo Básico Nacional, para su aplicación en la práctica pedagógica de las escuelas básicas.

En lo referido al rol de investigador, los docentes, en el proceso de planificación y ejecución de proyectos pedagógicos, toman en cuenta las interacciones del estudiante y les apoyan en las acciones de búsqueda, selección, procesamiento y aplicación de los referentes teóricos y prácticos requeridos para el cumplimiento de los objetivos que los orientan; esto evidencia, la acción desarrollada por los docentes en las escuelas básicas, busca apoyar al estudiante, lo que 


\section{Tabla 2}

\section{La gestión académico-administrativa desde la perspectiva de los docentes}

\begin{tabular}{lclcc}
\hline Gestión & \multicolumn{1}{c}{ Propiedades } & $\begin{array}{c}\mathbf{N}^{\circ} \\
\text { de Factores }\end{array}$ & $\begin{array}{c}\text { \% de Varianza } \\
\text { Explicada }\end{array}$ \\
\hline & 1 & $\begin{array}{l}\text { Roles del docente en interacciones } \\
\text { dinámicas de la praxis curricular }\end{array}$ & 4 & 58,808 \\
Académica & 2 & $\begin{array}{l}\text { Procesos del aprendizaje en la práctica } \\
\text { pedagógica }\end{array}$ & 2 & 45,017 \\
& 3 & Manejo de contenidos & 2 & 60,431 \\
& 4 & $\begin{array}{l}\text { Componentes curriculares que apoyan la } \\
\text { sistematización del proceso instruccional }\end{array}$ & 2 & 53,073 \\
& 5 & Lineamientos de apoyo al currículo & 1 & 46,741 \\
& 6 & $\begin{array}{l}\text { Socialización del currículo en los } \\
\text { procesos de escolarización }\end{array}$ & 1 & 56,698 \\
& 7 & Planificación & 1 & 37,711 \\
& 8 & Organización & 1 & 70,274 \\
& 9 & Dirección & 1 & 48,528 \\
& 10 & Evaluación & 1 & 58,267 \\
\hline
\end{tabular}

Fuente: Instrumento (A) aplicado a los docentes de aula.

hace manifiesto que se intenta asumen los lineamientos curriculares como elementos orientadores del proceso de formación y desarrollo de competencias en los alumnos.

En lo relacionado con el rol de promotor y gestor comunitario, se evidencian debilidades en la interacción que establece la escuela básica con la comunidad dado que, escasamente propicia la vinculación con esta última, en razón de sumar esfuerzos y voluntades en la formación y el desarrollo biopsicosocial-pedagógico de la población estudiantil. Al respecto los docentes en función corresponsable con el personal directivo, establecen una débil relación con los actores del ámbito externo a la escuela.
Se encontraron diferencias de opinión entre el personal directivo y docentes de aula, en cuanto a las oportunidades que ofrece la escuela a la comunidad en lo relacionado a estrategias y acciones de aprendizaje, asesorías e informaciones orientadas a la prevención y mejoramiento de sus condiciones de vida. Al respecto, según los docentes, no existe una programación sistemática desde la dirección del plantel hacia la búsqueda de salidas a problemas del contexto comunitario inmediato, esta situación tiende a reducir los logros en los procesos de la gestión académica y administrativa de la escuela básica; al desarrollar acciones socioeducativas aisladas de un entorno, que de alguna manera la condiciona, dada la inter- 
dependencia que se establece entre ambas instancias organizacionales.

Con respecto al rol de evaluador, destaca, de manera significativa, la preferencia del docente por la evaluación cuantitativa de carácter tradicional, situación que devela un fuerte arraigo hacia la aceptación de este tipo de evaluación. En este aspecto, la escala de valoración cuantitativa, representa el marco referencial para emitir juicios de valor al respecto.

El anclaje del docente, en este tipo de evaluación, constituye un obstáculo epistemológico para el abordaje, construcción y aplicación de la evaluación cualitativa, la cual emerge como requerimiento del Currículo Básico Nacional, sin embargo, la mayoría de los docentes no muestra dominio suficiente al respecto; además aducen que no es motivadora para el niño, los padres y representantes; porque los criterios y escalas de valoración en los que se sustenta, no les permiten apreciar, en su justo valor, los resultados de la misma. Los docentes cuestionan los cursos de capacitación que ofrece el Ministerio de Educación, Cultura y Deportes, porque se desarrollan en tiempos muy cortos, insuficientes para asimilar los elementos teóricos, metodológicos y procedimentales necesarios para la comprensión y aplicación del conocimiento sobre evaluación cualitativa.

Es generalizada la práctica unilateral en la evaluación, dado que no se socializan los resultados con los padres y representantes, para discutir y reflexionar de manera conjunta, sobre cada uno de los progresos y dificultades que presentan los alumnos en sus aprendizajes. Escasamente se visualiza una acción de intercambio sobre este proceso académi- co. Además, son pocos los padres y representantes que se preocupan y exigen explicaciones más detalladas sobre las fortalezas y debilidades del estudiante en su desempeño en el proceso escolar.

\section{La gestión académico-administrativa desde la perspectiva del personal directivo}

Al personal directivo le fue aplicado el instrumento "B". En este caso, se procedió de la misma forma que con los docentes (un análisis factorial por cada propiedad). Dicha información puede ser apreciada en la Tabla 3, se observa el número de factores y el porcentaje de varianza explicada que resultó del análisis factorial.

El análisis de las acciones desarrollas por el personal directivo en la gestión administrativa develan la existencia de escuelas que tienen personal directivo dotado de competencias para el desarrollo adecuado de los procesos inherentes a la gestión administrativa, haciendo posible la gestión académica a partir de la ejecución del currículo; propician condiciones en la planificación, organización, dirección y evaluación de proyectos en atención a los objetivos planteados; elementos éstos que hacen operativa y dinámica la gestión administrativa, según lo consideran Palladino y Palladino (1998:9) y Batanaz (1999:183).

Al confrontar estos resultados con los obtenidos en la información aportada por los docentes se observa cierta discrepancia; estos manifiestan que las acciones realizadas por algunos directivos no llenan las expectativas de los docentes, 


\section{Tabla 3}

\section{La gestión académico-administrativa desde la perspectiva del personal directivo}

\begin{tabular}{|c|c|c|c|c|}
\hline Gestión & & Propiedades & $\begin{array}{c}\mathrm{N}^{\circ} \\
\text { de Factores }\end{array}$ & $\begin{array}{c}\% \text { de Varianza } \\
\text { Explicada }\end{array}$ \\
\hline & 1 & $\begin{array}{l}\text { Roles del docente en Interacciones } \\
\text { dinámicas de la praxis curricular }\end{array}$ & 1 & 44,916 \\
\hline \multirow[t]{2}{*}{ Académica } & 2 & $\begin{array}{l}\text { Procesos del aprendizaje } \\
\text { en la práctica pedagógica } \\
\text { Componentes curriculares } \\
\text { que apoyan la sistematización } \\
\text { del proceso instruccional }\end{array}$ & 2 & 48,216 \\
\hline & 4 & $\begin{array}{l}\text { Socialización del currículo } \\
\text { en los procesos de }\end{array}$ & 2 & 49,946 \\
\hline \multirow[t]{5}{*}{ Administrativa } & 6 & escolarización & 1 & 39,956 \\
\hline & 7 & Planificación & 3 & 56,230 \\
\hline & 8 & Organización & 3 & 51,733 \\
\hline & 9 & Dirección & 3 & 61,348 \\
\hline & 10 & Evaluación & 2 & 52,221 \\
\hline
\end{tabular}

Fuente: Instrumento (B) aplicado al personal directivo.

dada la ausencia de diagnósticos de necesidades, tanto a nivel de la escuela como de la comunidad y, se adolece de programas coordinados intra y extra institucionales.

Con referencia al proceso de organización, los docentes, manifestaron que se carece de directivos con preparación adecuada para la gestión de los recursos humanos, para canalizar la instrumentación de las operaciones propias de la reforma curricular y del mantenimiento y coherencia del sistema escolar, en el cumplimiento puntual de los recaudos administrativos, agravado por la falta de personal obrero y de secretaría. Muchos docentes con funciones de director y subdirector están en calidad de encargados, por lo tanto no reciben el reconocimiento y la remuneración acorde a su desempeño.

En el proceso de dirección se evidencia que los proyectos son impuestos por el personal directivo como signo de autoritarismo, lo cual contradice el principio de gestión participativa planteado en la reforma curricular. Los procesos humanos de liderazgo y comunicación están ausentes, pues los conflictos no son manejados con estrategias y técnicas adecuadas, lo que confluye en problemas comunicacionales por el choque de opiniones, desintegración del personal (entre los dos turnos).

En el mismo orden de ideas, se contradicen docentes y directivos con los postulados de González (2000) y Silicio et al., (1999) ante la consideración de que 
el director de un centro educativo es el responsable en el liderazgo de acciones que incidan en el desempeño del personal con entusiasmo y energía; en el caso de este estudio se encontró un liderazgo débil, impositivo y lejano para impulsar cambios curriculares en la gestión académica y organizacionales en la gestión administrativa.

Con respecto a la evaluación de los procesos institucionales, los docentes insistieron en la ausencia de coordinación de la gestión académica y administrativa; aunado a la poca supervisión tanto del personal directivo como de las autoridades educativas regionales.

Estos resultados develan la necesidad de abordar nuevos estudios e investigaciones desde la totalidad del intercambio social en que se ubica la escuela básica, a partir de sus múltiples interrelaciones con el macro-contexto; asimismo, apuntan a la revisión exhaustiva de los procesos académicos y administrativos, de la Educación Básica con la intención de profundizar su estudio para mejorar y elevar la calidad de este nivel educativo, en un proceso dialéctico, que involucre a los distintos actores sociales hacia un proceso educativo inclusivo, enriquecedor y eficaz, que ofrezca múltiples oportunidades, en atención a los requerimientos y demandas de la población en el contexto social venezolano.

\section{Reflexiones finales}

Los resultados de la presente investigación apuntan hacia nuevas visiones y construcciones para mejorar y elevar la calidad del nivel de educación básica desde un proceso interactivo de discusión, refle- xiones y propuestas donde se involucren los distintos actores sociales para orientar un proceso educativo mas inclusivo, enriquecedor y eficaz, que ofrezca múltiples oportunidades a los miembros de la comunidad educativa para la participación, la toma de decisiones y la acción, para responder de manera adecuada a los requerimientos de la población en el contexto social venezolano.

Por otra parte, de los resultados deriva la necesidad de viabilizar los procesos de la gestión académica y administrativa a través de líneas de acción que permitan dar concreción a las innovaciones curriculares planteadas por el Estado para este nivel educativo para el desarrollo idóneo de los procesos escolares y el logro de la calidad, eficacia, pertinencia académica y social de las escuelas básicas venezolanas.

Los hallazgos generados en la investigación señalan, que existen diferencias en la estructura material, académica y administrativa en las escuelas básicas, de hecho, se observan instituciones educativas con alto y mediano desempeño en zonas urbanas, encontrándose en los espacios geográficos rurales y urbanos marginales escuelas cuyas carencias y limitaciones no le permiten acercarse a un desempeño aceptable; en ese sentido, las fortalezas encontradas en la realidad educativa de algunas escuelas básicas, pueden ser consolidadas a través de un adecuado provecho de la red de relaciones que se conjugan mediante los procesos curriculares y organizacionales las cuales pueden ser extendidas a la totalidad del contexto escolar y extraescolar. Mientras que en otras escuelas básicas es necesario subsanar las carencias y dificultades que presentan para que sus 
procesos académicos y administrativos sean atendidos y mejorados con mayores niveles de eficacia y eficiencia en beneficio de la población estudiantil.

\section{Referencias Bibliográficas}

Altuve, Magali (1997). Innovaciones educativas. Caracas-Venezuela: Universidad Pedagógica experimental Libertador.

Amarante, Ana (2000). Gestión directiva. Argentina. Magisterio del Río de la Plata.

Bar, Graciela (1999). Perfil y competencias del docente en el contexto institucional educativo. I Seminario-Taller sobre el perfil del docente y estrategias de formación. París: OEA. Perú

Batanaz, Luis (1999). Teoría y diseño curricular. México: Trillas

Bavaresco, de Prieto Aura M. (1997). Proceso Metodológico en la investigación. Tercera edición. Venezuela: Maracaibo. Ediluz.

Bounds, Gregory. Woods, Jhon (1999). Supervisión. México: Internacional Thomson

Bravo, Luis. Ramirez, Tulio y Pernía, Edgar. (2000). El sistema educativo venezolano. Reforma, descentralización y trabajo docente. Caracas: $\mathrm{Pa}$ napo.

Brown, Frederick (1996). Principios de la medición en psicología y educación. México: Editorial El Manual Moderno S.A.

Contreras, Julio (1995). La invetigación en la acción. ¿Qué es?. Cuadernos de Pedagogía. Sevilla: Diada

Corrales, Olga. Jimenez, María (1994). El docente y la investigación. Revista de Educación. Vol 18, № 2. Editorial de la Universidad de Costa Rica.
De La Garza, Jorge (1995). Análisis de la información mercadológica a través de la estadística multivariante. México: Editorial Alambra.

Delors, Jacques (1996). La educación encierra un tesoro. Madrid: SAntillana. UNESCO

Diez De Castro, Emilio. Garcïa, Julio. Martïn, Francisca. Pianez C. Rafael (2001). Administración y Dirección. España: Mc Graw Hill

Escalona, María; Nava, Fredefinda; Luque, Rafael; Rios, Yaneth; Vilchez, Angel. (2000). Situaciones cognitivas, creencias y resolución de problemas matemáticos. Revista en Encuentro Educacional Vol 7, № 1. Maracaibo: Universidad del Zulia.

Florez O. Rafael (1999). Evaluación, Pedagogía y Cognición. Colombia: Mc Graw Hill.

García, Filomena (1997). Organización escolar y gestión de centros educativos. Málaga: Algibe

González, Marcos (2000). Administración escolar. México: Castillo.

Hernández, Acacia. (1994). Investigación como diseño. Tesis Doctoral. Venezuela: Caracas.

Hernández, Sampieri Roberto; Fernández, Collado Carlos y Baptista, Lucio Pilar (1994). Metodología de la investigación. Colombia: Mc Graw Hill.

Hernández, Sampieri Roberto; Fernández, Collado Carlos y Baptista, Lucio Pilar (2000). Metodología de la investigación. Colombia: Mc Graw Hill.

Lepeley, María (2001).Gestión y calidad en educación. Chile: Mc Graw Hill

Palladino, Enrique y Palladino Leandro (1998) Administración Organizacional. Argentina: Espacio.

Perez, Carlota. (2000). La reforma educativa ante el cambio de paradigma. Caracas: Eureka. 
Romero, Francisco (1996). La cultura organizativa y la gestión administrativa en los centros educativos. Revista Tarbiya № 13. España: Universidad autónoma de Madrid.

Secretaría de Educación del Estado Zulia (2003). Maracaibo. Mimeografiado.

Sierra, Restituto (1981). Ciencias Sociales. Análisis estadístico y modelos matemáticos. Teorías y ejercicios. España: PARANINFO

Silicio, Alfonso, Casares, David y González, José Luis. (1999). Liderazgo, valo- res y cultura organizacional hacia una organización competitiva. España. Editorial Escuela Española.

UNESCO (1996). Estudios y documentos de educación № 39. La autogestión en los sistemas educativos. Francia.

Vayer, Pierre, Duval, Amand, Roncin, Charles (1993). Una ecología de la escuela. Barcelona-España: Paidós.

Wilson, John (1992). Cómo valorar la calidad de la enseñanza. Madrid: Paidós.

Zona Educativa Zulia (2003). Distribución de Escuelas Básicas por Municipio. Maracaibo. Mimeografiado. 